\title{
Exposition aux dispositifs d'éclairage scénique : risque pour la santé des professionnels du spectacle vivant ou enregistré
}

\author{
S. SALSI, A. BARLIER-SALSI
}

(Manuscript reçu le 19 novembre 2012, accepté le 8 avril 2013)

RÉSUMÉ La directive européenne 2006/25/CE, relative à l'exposition des travailleurs aux rayonnements optiques, prévoit l'évaluation des risques des situations de travail. Elle est particulièrement importante dans l'industrie des arts du spectacle où des expositions intentionnelles à des projecteurs peuvent être exigées pendant des périodes de l'ordre de 8 heures par jour. L'objectif de la présente étude était de fournir aux éclairagistes des informations, relatives aux risques associés aux projecteurs, afin de les aider à évaluer les risques a priori d'un plan lumière. Elle a consisté à déterminer les risques de 63 projecteurs différents puis à les répartir dans les 4 groupes de risques définis par la norme EN 62471, à calculer leur distance minimale d'exposition admissible, correspondant à l'utilisation de 1 et de 5 projecteurs pour une durée d'exposition journalière de 8 heures. Cette étude a mis en évidence que les projecteurs sont susceptibles de poser des problèmes sanitaires pour les salariés des arts du spectacle. Le schéma de classification proposé par la norme EN 62471 n'est pas suffisant pour évaluer les risques d'un plan lumière. De plus, la distance d'exposition et la durée d'exposition ne constituent pas des paramètres pertinents pour réduire les risques à des valeurs admissibles.

ABSTRACT Exposure to scenic lighting devices: risk to the health of entertainment professionals.

The European directive 2006/25/EC on minimum health and safety requirements regarding the exposure of workers to the risks arising from optical radiation provides for risk assessment in workplaces. This is particularly important in entertainment, where intentional exposure to spotlights may be required for periods of 8 hours per working day. The aim of the study was to provide the lighting engineers with information, relative to the risks associated with spotlights, to help them to estimate the risks a priori of a lighting plan. This study consisted of determining the risks associated with 63 different spotlights, then distributing them into 4 groups of risks defined by the standard EN 62471, in calculating their permissible minimal exposure distance, corresponding to the use of 1 and 5 spotlights for a daily exposure time of 8 hours. This study highlighted that spotlights may cause health problems for entertainment workers. The classification proposed by the standard EN 62471 is not sufficient to estimate the risks of a lighting plan. Furthermore, the permissible minimal exposure distance and the permissible maximal exposure time do not constitute relevant parameters to reduce the risks to acceptable values.

Keywords: optical radiation / entertainment / spotlight / risk / European directive

INRS, Département Ingénierie des Equipements de Travail, 1 rue du Morvan, CS 60027, 54519 Vandoeuvre Cedex, France. 


\section{Introduction}

Le domaine d'application de la directive européenne 2006/25/CE (Parlement européen, 2006), relative à l'exposition des travailleurs aux rayonnements optiques artificiels (ROA), concerne toute source de ROA cohérente ou incohérente. Cependant, le présent article ne traite que des sources de ROA incohérents. Afin de protéger les travailleurs contre les risques dus aux ROA, la directive européenne définit des grandeurs énergétiques à déterminer dans différents domaines spectraux, de longueurs d'onde comprises entre 180 et $3000 \mathrm{~nm}$, et spécifie les valeurs limites d'exposition (VLE) correspondantes. Ces VLE sont issues des recommandations de l'ICNIRP $^{1}$ (ICNIRP, 1997; 2004). Ces dispositions règlementaires sont particulièrement importantes pour l'industrie des arts du spectacle $^{2}$ où, du fait de contingences opérationnelles, des expositions intentionnelles à des projecteurs d'éclairage scénique peuvent être exigées pendant des périodes de l'ordre de 8 heures par jour (O'Hagan et al., 2011).

En effet, dans ce domaine d'activité, les principales règles d'esthétiques auxquelles l'éclairage scénique obéit sont les suivantes :

- rendre visible la scène de façon réaliste et/ou naturelle et favoriser les éléments des décors ;

- créer l'atmosphère du spectacle en éclairant les artistes et les décors en fonction des moments choisis. Selon l'atmosphère souhaitée, les projecteurs sont équipés soit de lampes « tungstène halogène » $(\mathrm{TH})$ de température de couleur de l'ordre de 3000 à $3200 \mathrm{~K}$, soit de lampes aux « halogénures métalliques $»^{3}$ (HMI) dont la température de couleur est proche de celle de la lumière du jour, typiquement $5600 \mathrm{~K}$.

Selon le type de spectacle, ces règles conduisent l'éclairagiste à mettre en œuvre plusieurs centaines de projecteurs, de puissances diverses, programmés pour fonctionner à des moments particuliers et pendant des durées déterminées. À un spectacle donné correspond donc un «plan lumière » spécifique (type, nombre et puissance des projecteurs, positions sur la scène et dans la salle, durées et périodes d'allumage). L'exposition énergétique des artistes n'est donc pertinente que pour le spectacle concerné, elle ne peut en aucun cas être extrapolée à d'autres «plans lumière ». C'est pour ces raisons que les données disponibles dans la littérature sont peu ou prou utilisables, car elles sont caractéristiques de conditions

\footnotetext{
ICNIRP : International commission on non-ionizing radiation protection.

2 En présence de public, on parle de « spectacle vivant » (théâtre, danse, concert, ...), en revanche, lorsqu'il s'agit de réaliser des produits télévisuels ou cinématographiques et de les diffuser par l'intermédiaire d'un support alors on parle de « spectacle enregistré ».

3 Lampe à décharge haute pression. Ces lampes sont généralement appelées lampes MSR, acronyme de «medium source rare earth gaz » ou HMI pour « hydrargyrum mittlere bongenlängen IOD »,
} 
d'utilisation spécifiques ; elles sont donc difficilement transposables. En effet, une première étude (Hietanen et Hoikkala, 1990) réalisée sur 11 projecteurs, équipés de lampes TH ou de lampes HMI, a montré que la vision directe de ces projecteurs (risque dû à la lumière bleue) doit être limitée, selon leur puissance, de 10 min à $3 \mathrm{~h}$. De plus, les durées maximales d'exposition journalières admissibles au rayonnement ultraviolet (UV) produit, à des distances comprises entre $2,25 \mathrm{~m}$ et $10 \mathrm{~m}$, varient de $5 \mathrm{~min}$ à $8 \mathrm{~h}$. D'une seconde étude (O'Hagan et al., 2011), réalisée sur 6 projecteurs équipés de lampes HMI, il ressort que les durées d'exposition maximales journalière admissibles au rayonnement ultraviolet, produit par les projecteurs à $1 \mathrm{~m}$, varient de $11 \mathrm{~s}$ à $4 \mathrm{~h}$ selon leur puissance. Ces 2 études ont montré que les dispositifs d'éclairage scéniques sont susceptibles de présenter des risques, dans certaines conditions d'utilisation, dans les domaines UV et de la lumière bleue du spectre. Cependant, aucune n'a évalué les risques inhérents au rayonnement infrarouge (IR) qu'ils produisent.

Il est néanmoins nécessaire de fournir aux professionnels du spectacle des informations relatives aux risques associés aux projecteurs scéniques indépendantes de leurs conditions d'utilisation. Ces informations sont indispensables aux éclairagistes pour les aider à évaluer les risques a priori d'un «plan lumière ».

La norme EN 62471 (2008) permet de répondre à cette problématique. Elle s'applique aux sources incohérentes de ROA, à spectre large, alimentées électriquement, y compris les LEDs, à l'exclusion des lasers, dans un domaine de longueurs d'onde comprises entre 200 et $3000 \mathrm{~nm}$. Les limites d'exposition (LE) spécifiées par cette norme se réfèrent aux VLE de la directive européenne. Un élément important de cette norme est le schéma de classification traduisant les risques photobiologiques produits. Quatre groupes de risques sont identifiés :

- groupe de risque $0, \mathrm{GR}(0)$ : sans risque ;

- groupe de risque $1, \mathrm{GR}(1)$ : faible risque ;

- groupe de risque $2, \mathrm{GR}(2)$ : risque modéré ;

- groupe de risque $3, \mathrm{GR}(3)$ : risque élevé.

Toutefois, cette classification s'applique à un seul et unique appareil. Ainsi, un projecteur d'un type et d'une puissance donnés appartenant au groupe de risque $\mathrm{GR}(0)$, ne présente aucun risque particulier quelles que soient ses conditions d'utilisation. En revanche, l'utilisation de 5 projecteurs répertoriés $\mathrm{GR}(0)$ de même type et de même puissance peut produire des risques non négligeables. Il convient donc de donner des informations sur les modifications des niveaux de risques consécutives à l'utilisation de plusieurs projecteurs de même type.

L'étude présentée ci-après a donc consisté à évaluer les risques associés à 63 projecteurs différents puis à les répartir dans les 4 groupes de risques définis par 
TABLEAU I

Liste des projecteurs mesurés.

List of measured spotlights.

\begin{tabular}{cccc}
\hline Type de projecteur & Type de lampe & Nombre & Puissance (W) \\
\hline \multirow{2}{*}{ PAR } & TH & 4 & $575-1000$ \\
\cline { 2 - 4 } & HMI & 14 & $575-12000$ \\
\multirow{2}{*}{ FRESNEL } & TH & 19 & $300-10000$ \\
\cline { 2 - 4 } & HMI & 26 & $575-12000$ \\
\hline
\end{tabular}

la norme EN 62471 et enfin à calculer leur distance minimale d'exposition admissible respective $\left(\mathrm{D}_{\text {min-exp }}\right)$, distance au-delà de laquelle il n'existe plus aucun risque, correspondant à l'utilisation de 1 et de 5 projecteurs de même type et de même puissance, pour une durée d'exposition journalière arbitraire de $8 \mathrm{~h}$.

\section{Matériel et méthode}

\subsection{Différents types de projecteurs étudiés}

Des professionnels du spectacle vivant et enregistré (producteurs de spectacles et de cinéma, fabricants, Opéra National de Paris) ont sélectionné, parmi les projecteurs qu'ils utilisent le plus fréquemment, puis mis à notre disposition 18 projecteurs à réflecteur parabolique (PAR) et 45 à lentille de Fresnel (Fresnel) de différentes puissances. Ces projecteurs étaient équipés de lampes TH ou HMI et se répartissaient comme indiqué au tableau I.

\subsection{Classification, en termes de risque, des projecteurs selon la norme EN 62471}

Le schéma de classification proposé par la norme EN 62471 ne prend pas en compte tous les risques inhérents à l'exposition aux ROA sur l'intervalle 200-3000 nm. Seuls ceux présentés dans le tableau II, en fonction du domaine spectral concerné, sont pris en considération. Dans ce tableau, $\mathrm{t}_{\max }$ représente la durée maximale d'exposition journalière admissible spécifiée par la norme pour un domaine spectral $[\lambda 1, \lambda 2]$ et un groupe de risque $\mathrm{GR}(\mathrm{x})$ comme indiqué dans le tableau III ; $t_{\max }$ détermine les LE correspondantes (Tabs. II et IV). Les terminologies des grandeurs utilisées dans cet article sont détaillées en annexe.

Un projecteur est classé dans un $\mathrm{GR}(\mathrm{x})$ si et seulement si, dans tous les domaines spectraux $[\lambda 1, \lambda 2]$, son émission énergétique, caractérisée par $\mathrm{E}_{\mathrm{s}}, \mathrm{E}_{\mathrm{UVA}}$, $\mathrm{L}_{\mathrm{B}}, \mathrm{L}_{\mathrm{R}}$ et $\mathrm{E}_{\mathrm{IR}}$, est inférieure à la $\mathrm{LE}_{[\lambda 1, \lambda 2]}$ correspondantes données dans le tableau IV ; sinon, il est classé dans le GR(x+1) immédiatement supérieur. 
TABLEAU II

Résumé des risques et des LE pris en compte par la norme EN 62471.

Summary of the risks and of the LE taken into account by the standard EN 62471.

\begin{tabular}{|c|c|c|c|c|}
\hline Identification des risques & $\begin{array}{c}\text { Organe } \\
\text { cible }\end{array}$ & Domaine spectral & $\begin{array}{c}\text { Grandeurs } \\
\text { énergétiques }\end{array}$ & LE \\
\hline Kératite, conjonctivite & œil & \multirow{2}{*}{$\begin{array}{l}200-400 \mathrm{~nm} \\
\left(\mathrm{UV}_{\text {Actinique }}\right)\end{array}$} & \multirow{2}{*}{$\mathrm{E}_{\mathrm{S}}$} & $30 / t_{\max }$ \\
\hline Erythème, cancers & peau & & & $\left(\mathrm{W} \cdot \mathrm{m}^{-2}\right)$ \\
\hline Cataractes & œil & $\begin{array}{c}315-400 \mathrm{~nm} \\
\left(\mathrm{UV}_{\mathrm{A}}\right)\end{array}$ & $\mathrm{E}_{\mathrm{UVA}}$ & $\begin{array}{c}10000 / \mathrm{t}_{\max } \\
\left(\mathrm{W} \cdot \mathrm{m}^{-2}\right)\end{array}$ \\
\hline $\begin{array}{l}\text { Lésion photochimique } \\
\text { rétinienne }\end{array}$ & œil & $\begin{array}{c}300-700 \mathrm{~nm} \\
\text { (lumière bleue) }\end{array}$ & $\mathrm{L}_{\mathrm{B}}$ & $\begin{array}{c}10^{6 / \mathrm{t}_{\max }} \\
\left(\mathrm{W} \cdot \mathrm{m}^{-2} \cdot \mathrm{sr}^{-1}\right)\end{array}$ \\
\hline Lésions thermiques rétiniennes & œil & $\begin{array}{c}380-1400 \mathrm{~nm} \\
\left(\text { Visible }+\mathrm{IR}_{\mathrm{A}}\right)\end{array}$ & $\mathrm{L}_{\mathrm{R}}$ & $\begin{array}{c}\frac{50000}{\alpha_{\text {projecteur }} \cdot{ }_{\text {max }} 0,25} \\
\left(\mathrm{~W} \cdot \mathrm{m}^{-2} \cdot \mathrm{sr}^{-1}\right)\end{array}$ \\
\hline $\begin{array}{l}\text { Lésions thermiques } \\
\text { cornéennes, cataractes }\end{array}$ & œil & $\begin{array}{l}780-3000 \mathrm{~nm} \\
\left(\mathrm{IR}_{\mathrm{A}}+\mathrm{IR}_{\mathrm{B}}\right)\end{array}$ & $\mathrm{E}_{\mathrm{IR}}$ & $\begin{array}{c}18000 / \mathrm{t}_{\max }{ }^{0,75} \\
\left(\mathrm{~W} \cdot \mathrm{m}^{-2}\right)\end{array}$ \\
\hline
\end{tabular}

TABLEAU III

Grandeurs calculées et $t_{\max }$ relatives aux 3 groupes de risques proposés par la norme EN 62471 . Calculated quantities and $t_{\text {max }}$ relative to the 3 groups of risks proposed by the standard EN 62471 .

\begin{tabular}{|c|c|c|c|c|c|}
\hline \multirow{2}{*}{$\begin{array}{l}\text { Domaine } \\
\text { spectral }\end{array}$} & \multirow{2}{*}{ Grandeurs énergétiques calculées } & \multicolumn{4}{|c|}{$t_{\max }$ en $s$} \\
\hline & & $\mathrm{GR}(0)$ & GR(1) & GR(2) & GR(3) \\
\hline $\mathrm{UV}_{\text {Actinique }}$ & $E_{s}=\sum_{200}^{400} E_{\lambda(0,20 m)} \cdot S(\lambda) \cdot \Delta \lambda$ & 28800 & 10000 & 1000 & \multirow{5}{*}{$\begin{array}{l}\text { Regroupe les } \\
\text { projecteurs } \\
\text { dont } \\
\text { l'émission } \\
\text { énergétique } \\
\text { excède les LE } \\
\text { du groupe de } \\
\text { risque GR(2) }\end{array}$} \\
\hline $\mathrm{UV}_{\mathrm{A}}$ & $E_{U V A}=\sum_{315}^{400} E_{\lambda(0,20 m)} \cdot \Delta \lambda$ & 1000 & 300 & 100 & \\
\hline Lumière bleue & $L_{B}=\sum_{300}^{700} L_{\lambda} \cdot B(\lambda) \cdot \Delta \lambda$ & 10000 & 100 & 0,25 & \\
\hline Visible $+\mathrm{IR}_{\mathrm{A}}$ & $L_{R}=\sum_{380}^{1400} L_{\lambda} \cdot R(\lambda) \cdot \Delta \lambda$ & 10 & 10 & 0,25 & \\
\hline $\mathrm{IR}_{\mathrm{A}}+\mathrm{IR}_{\mathrm{B}}$ & $E_{I R}=\sum_{780}^{3000} E_{\lambda(0,20 \mathrm{~m})} \cdot \Delta \lambda$ & 1000 & 100 & 10 & \\
\hline
\end{tabular}

TABLEAU IV

Limites d'exposition, relatives aux différentes $t_{\text {max }}$, fixées par la norme pour les 3 GR(x). Exposure limits, relative to the different $t_{\text {max }}$, fixed by the standard for the 3 GR(x).

\begin{tabular}{ccccc}
\hline $\begin{array}{c}\text { Domaine spectral } \\
{[\lambda 1, \lambda 2]}\end{array}$ & $\mathrm{GR}(0)$ & $\mathrm{GR}(1)$ & $\mathrm{GR}(2)$ & $\mathrm{Unités}$ \\
\cline { 2 - 5 } & 0,001 & 0,003 & 0,03 & $\mathrm{~W} \cdot \mathrm{m}^{-2}$ \\
\hline $\mathrm{UV}_{\text {Actinique }}$ & 10 & 33 & 100 & $\mathrm{~W} \cdot \mathrm{m}^{-2}$ \\
$\mathrm{UV}_{\mathrm{A}}$ & 100 & 10000 & 4000000 & $\mathrm{~W} \cdot \mathrm{m}^{-2} \cdot \mathrm{sr}^{-1}$ \\
Lumière bleue & $2800 / \alpha_{\text {projecteur }}$ & $2800 / \alpha_{\text {projecteur }}$ & $71000 / \alpha_{\text {projecteur }}$ & $\mathrm{W} \cdot \mathrm{m}^{-2} \cdot \mathrm{sr}^{-1}$ \\
Visible $+\mathrm{IR}_{\mathrm{A}}$ & 100 & 570 & 3200 & $\mathrm{~W} \cdot \mathrm{m}^{-2}$ \\
\hline $\mathrm{IR}_{\mathrm{A}}+\mathrm{IR}_{\mathrm{B}}$ & & & & \\
\hline
\end{tabular}




\subsection{Détermination des grandeurs énergétiques $E_{s}, E_{U V A}, L_{B}, L_{R}$ et $E_{I R}$ définies dans le tableau III}

\subsubsection{Détermination de $E_{s}, E_{U V A}$, et $E_{I R}$}

Les grandeurs énergétiques $\mathrm{E}_{\mathrm{S}}, \mathrm{E}_{\mathrm{UVA}}, \mathrm{E}_{\mathrm{IR}}$, ont été déterminées à partir de $\mathrm{E}_{\lambda(0,20 \mathrm{~m})}$. En effet, la norme prévoit de relever $\mathrm{E}_{\lambda(0,20 \mathrm{~m})}$, sur l'ensemble du spectre des rayonnements optiques (200-3000 nm), dans un plan situé à une distance de $0,20 \mathrm{~m}$ du projecteur considéré selon la direction normale à sa surface d'émission. Or compte tenu, d'une part du rayonnement intense produit par ces appareils, d'autre part de la sensibilité du spectroradiomètre utilisé, $\mathrm{E}_{\lambda \text { (mesure) }}$ a été relevé, selon la procédure définie par la norme EN 62471, à des distances variant de 0,60 m à 8,00 m selon la puissance des projecteurs. Ces mesures ont été réalisées en laboratoire selon les conditions normales d'utilisation des projecteurs, à savoir : alimentation par le secteur et à pleine puissance afin de déterminer leurs risques maximum. Ces relevés ont été effectués à l'aide d'un spectroradiomètre à balayage OPTRONIC - OL 750, équipé d'une sphère d'intégration, calibré en éclairements énergétiques spectriques à partir d'étalons secondaires eux-mêmes étalonnés par rapport aux étalons du NIST $^{4}$ avec une précision variant de 2 à $6 \%$ selon les longueurs d'onde.

Dans ces conditions, si $E_{\lambda(\text { mesure) }}, F_{\text {config(mesure) }}, E_{\lambda(0,20 \mathrm{~m})}$ et $F_{\text {config }(0,20 \mathrm{~m})}$ sont, respectivement, l'éclairement énergétique spectrique et le facteur de configuration du projecteur correspondant aux conditions de mesures et à une distance de $0,20 \mathrm{~m}$, alors $\mathrm{E}_{\lambda(0,20 \mathrm{~m})}$ a pour expression :

$$
E_{\lambda(0,20 \mathrm{~m})}=E_{\lambda(\text { mesure })} \frac{F_{\text {config }(0,20 \mathrm{~m})}}{F_{\text {config }(\text { mesure })}} .
$$

Ces calculs ont été réalisés, sur l'ensemble des 63 projecteurs étudiés, à l'aide du logiciel « CatRayon ${ }^{\circledR}{ }^{5}$ (CatRayon version 33 ou version supérieure) développé par l'INRS (Barlier-Salsi et Salsi, 1996 ; 2007a ; 2007b ; INRS, 2012).

\subsubsection{Détermination de $L_{B}$ et $L_{R}$}

Les luminances énergétiques pondérées $\mathrm{L}_{B}$ et $\mathrm{L}_{\mathrm{R}}$ ont été déduites des mesures de $\mathrm{E}_{\lambda(0,20 \mathrm{~m})}$, de la luminance lumineuse moyenne $\left(\mathrm{L}_{\mathrm{v}}\right)$ des projecteurs et de l'éclairement lumineux $\mathrm{E}_{\mathrm{v}(0,20 \mathrm{~m})}$ qu'ils produisent à $0,20 \mathrm{~m}$, comme suit :

$$
L_{B}=\frac{L_{v}}{E_{v(0,20 m)}} \sum_{300}^{700} E_{\lambda(0,20 \mathrm{~m})} B(\lambda) \Delta \lambda \quad L_{R}=\frac{L_{v}}{E_{v(0,20 \mathrm{~m})}} \sum_{380}^{1400} E_{\lambda(0,20 \mathrm{~m})} R(\lambda) \Delta \lambda .
$$

4 National institute of standards and technologies.

5 CatRayon ${ }^{\circledR}$ est un outil logiciel qui permet d'évaluer, conformément à la directive européenne, l'exposition des salariés à différentes sources de ROA pour différentes positions des sources ou des opérateurs et diverses durées d'exposition. Il détermine en outre les moyens de protection collective et individuelle à mettre en œuvre. Ces évaluations sont réalisables soit à partir des caractéristiques géométriques et spectrales des sources et des filtres de protection contenus dans ses bases de données, soit à partir des caractéristiques des sources mesurées in situ. 


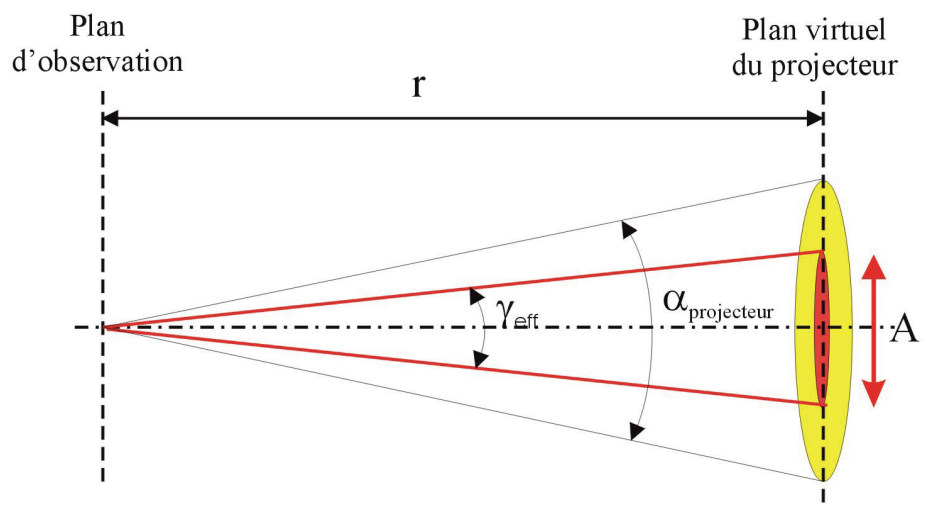

Figure 1 - Conditions d'observation des projecteurs pour l'évaluation de la luminance énergétique selon la norme EN 62471.

Les paramètres suivants doivent être définis pour l'évaluation des risques :

- $\boldsymbol{r}$ : distance d'observation ;

- $\alpha_{\text {projecteur }}:$ dimension angulaire du projecteur vue de l'observateur ;

- $\gamma_{\text {eff }}$ : champ de vision effectif

- A : surface effective d'intégration de la luminance lumineuse du projecteur.

Observation conditions of spotlights for the evaluation of the radiance according to the standard EN 62471.

The following parameters must be defined for the risk assessment:

- r: observation distance;

- $\alpha$ projecteur: angular dimension of the luminaire seen by the viewer;

- $\gamma_{\text {eff: }}$ : angle of acceptance;

- A: effective surface of integration of the luminaire radiance.

Tous ces calculs ont été réalisés comme précédemment à l'aide du logiciel «CatRayon ${ }^{\circledR}{ }$.

Afin de tenir compte des mouvements oculaires, consécutifs à la fixation du regard, la norme EN 62471 prévoit de mesurer la luminance énergétique spectrique «moyenne » avec un champ de vision effectif $\gamma_{\text {eff }}$ (angle solide effectif sous lequel l'observateur voit la source) compris entre 1,7 et $100 \mathrm{mrad}$ selon les durées d'exposition journalière maximales admissibles $\left(\mathrm{t}_{\max }\right)$ correspondant aux différents groupes de risque ( $c f$. Tabs. III et IV). En effet, les mouvements oculaires ont un impact majeur sur les risques oculaire associés aux domaines visible et proche infrarouge du spectre. Ils augmentent la surface irradiée de la rétine et leur amplitude dépend directement de la durée d'observation. De ce fait, $\gamma_{\text {eff }}$ et la surface effective de la source A (surface utilisée pour l'intégration de la luminance énergétique spectrique) varient avec le temps d'observation (Schulmeister, 2001 ; 2011) (cf. Fig. 1). 


\section{TABLEAU V}

Champ de vision effectif $\gamma_{\text {eff }}$ en fonction de $t_{\max }$ pris en compte dans le domaine visible, par la norme EN 62471 pour la détermination de luminance énergétique des projecteurs.

Angle of acceptance $\gamma_{e f f}$ according to $t_{\max }$ taken into account in the visible domain, by the standard EN 62471 for the determination of spotlight radiance.

\begin{tabular}{ccc}
\hline Identification du risque & Groupe de risque & $\begin{array}{c}\text { Champ de vision effectif } \\
\gamma_{\text {eff }}(\text { rad })\end{array}$ \\
\hline \multirow{2}{*}{ Lumière bleue } & GR(0) & 0,1 \\
& GR(1) & 0,0011 \\
Lésions thermiques rétiniennes & GR(2) & 0,0017 \\
& GR(0) & 0,011 \\
& GR(1) & 0,011 \\
\hline
\end{tabular}

Ainsi $\mathrm{L}_{\mathrm{v}}$ est la luminance lumineuse moyenne, mesurée sur la surface d'émission A ( $c f$. Fig. 1), correspondant au champ de vision effectif $\gamma_{\text {eff }}$; lequel varie avec $\mathrm{t}_{\max }$ comme spécifié par la norme pour les différents $\mathrm{GR}(\mathrm{x})(c f$. Tab. V).

Lorsque $\gamma_{e f f}$ est plus grand que $\alpha_{\text {projecteur }}$, il convient de moyenner la luminance lumineuse sur une surface effective plus grande que la surface d'émission du projecteur. La luminance résultante, à prendre en compte dans l'évaluation de risque, est donc inférieure à la luminance moyenne du projecteur (CEI/TR 624712, 2009).

Quelle que soit la taille de la surface d'émission du projecteur, $\gamma_{\text {eff }}$ ne peut être supérieur à $0,1 \mathrm{rad}$, angle correspondant à $\mathrm{t}_{\max }>10000 \mathrm{~s}$.

\subsubsection{Mesure de la luminance lumineuse des projecteurs correspondant aux différents champs de vision effectifs $\gamma_{\text {eff }}$}

La luminance lumineuse des projecteurs a été mesurée à l'aide d'un vidéophotomètre TECHNO-TEAM LMK 98-2. Il s'agit d'un photomètre d'imagerie numérique constitué d'une caméra 12 bits, à capteur CCD 2/3', de résolution $1300 \times 1030$ pixels, et d'un logiciel d'analyse d'images. Cet ensemble permet, à partir d'images de la surface d'émission des projecteurs, de déterminer les $\mathrm{L}_{\mathrm{v}}$ correspondant aux différents $\gamma_{\text {eff }}$ spécifiés par la norme (cf. Fig. 2).

\subsection{Détermination des distances minimales d'exposition admissibles correspondant à l'utilisation de 1 et de 5 projecteurs de même type et de même puissance}

La distance minimale d'exposition admissible $\left(\mathrm{D}_{\text {min-exp }}\right)$ d'un projecteur, distance au-delà de laquelle aucun risque ne subsiste, correspond à la plus grande des 


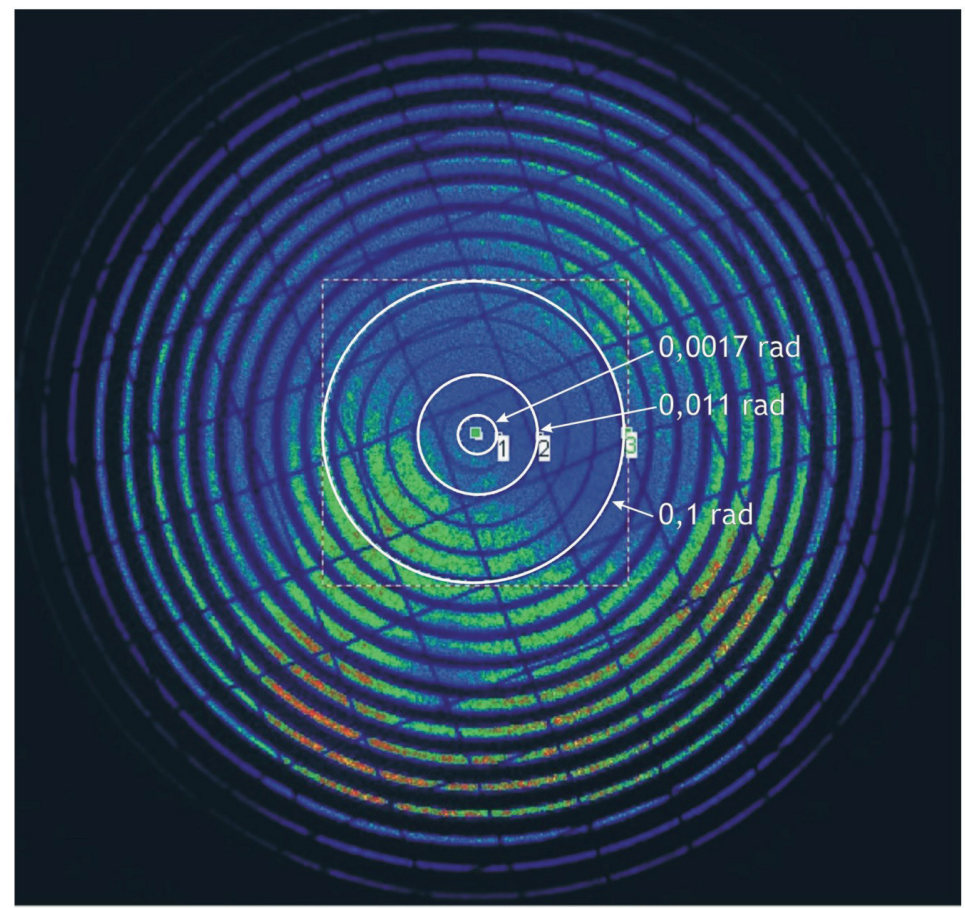

Figure 2 - Image de la surface d'émission d'un projecteur.

Image of a luminaire emission surface.

distances minimales d'exposition admissible $\left(D_{\min -\exp }\left[\lambda 1, \lambda_{2}\right]\right)$ déterminées pour chacun des domaines spectraux pris en compte.

Les risques associés aux projecteurs dans les différents domaines spectraux $[\lambda 1, \lambda 2]$ et correspondant à une distance d'exposition de $0,20 \mathrm{~m}$ et une durée d'exposition journalière de $8 \mathrm{~h}$, ont été calculés à l'aide de «CatRayon ${ }^{\circledR}$ » en termes « d'indice de risque ${ }^{6}\left(\mathrm{I}_{(0,20 \mathrm{~m}[\lambda 1, \lambda 2])}\right)$.

Donc $\mathrm{D}_{\min -\exp [\lambda 1, \lambda 2]}$ est, pour chaque domaine spectral $[\lambda 1, \lambda 2]$, la distance telle que :

$$
I_{(\mathrm{d}>\text { Dmin-exp}[\lambda 1, \lambda 2])}=1
$$

\footnotetext{
6 L'indice de risque $\mathrm{I}_{[\lambda 1, \lambda 2]}$ relatif à un domaine spectral donné $[\lambda 1, \lambda 2]$, se définit par le rapport de l'émission énergétique des projecteurs, objectivée par les grandeurs $\mathrm{E}_{\mathrm{S}}, \mathrm{E}_{\mathrm{UVA}}, \mathrm{L}_{\mathrm{B}}, \mathrm{L}_{\mathrm{R}}$ et $\mathrm{E}_{\mathrm{IR}}$, à la $\mathrm{LE}$ correspondantes. Ainsi, $\mathrm{I}_{[\lambda 1, \lambda 2]}>1$ signifie que la $\mathrm{LE}$ relative au domaine spectral $[\lambda 1, \lambda 2]$ est dépassée et qu'un risque existe.
} 


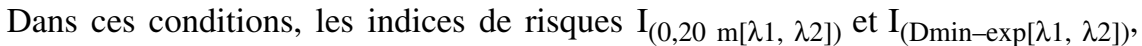
correspondant au domaine spectral $[\lambda 1, \lambda 2]$ et, respectivement, aux distances d'exposition $0,20 \mathrm{~m}$ et $\mathrm{D}_{\text {min-exp}}[\lambda 1, \lambda 2]$ ont pour expressions :

$$
I_{(0,20 m[\lambda 1, \lambda 2])}=\frac{F_{\text {config }(0,20 m)} L e_{[\lambda 1, \lambda 2]}}{L E_{[\lambda 1, \lambda 2]}}
$$

et

$$
I_{(\operatorname{Dmin}-\exp [\lambda 1, \lambda 2])}=\frac{F_{\operatorname{config}\left(D_{\min -\exp [\lambda 1, \lambda 2]}\right)} L e_{[\lambda 1, \lambda 2]}}{L E_{[\lambda 1, \lambda 2]}}
$$

où $\mathrm{F}_{\text {config }(0,20 \mathrm{~m})}, \mathrm{F}_{\text {config(Dmin-exp })}$ et $\mathrm{Le}_{[\lambda 1, \lambda 2]}$ sont respectivement les facteurs de configuration du projecteur considéré correspondant aux distances d'exposition $0,20 \mathrm{~m}$ et $D_{\min -\exp [\lambda 1, \lambda 2]}$ et sa luminance énergétique dans le domaine spectral $[\lambda 1, \lambda 2]$.

Comme les surfaces d'émission de tous les projecteurs étudiés ont une forme circulaire (de rayon $\mathrm{R}$ ) alors :

et

$$
\begin{gathered}
F_{\text {config }(0,20 m)}=\left[\frac{\pi R^{2}}{R^{2}+0,20^{2}}\right] \\
F_{\text {config }\left(D_{\min -\exp [\lambda 1, \lambda 2]}\right)}=\left[\frac{\pi R^{2}}{R^{2}+D_{\min -\exp [\lambda 1, \lambda 2]}}\right] .
\end{gathered}
$$

La distance minimale d'exposition admissible $\left(D_{\min -\exp \left[\lambda 1, \lambda_{2}\right]}\right)$, relative au domaine spectral $[\lambda 1, \lambda 2]$ se déduit des relations (1), (2), (3) et (4) comme suit :

$$
D_{\min -\exp [\lambda 1, \lambda 2]}=\left[I_{0,2[\lambda 1, \lambda 2]}\left(R^{2}+0,20^{2}\right)-R^{2}\right]^{0,5} .
$$

Par suite, la $D_{\text {min-exp }}$ d'un projecteur se déduit de la relation suivante :

$$
D_{\text {min-exp }} \geq M A X_{\forall[\lambda 1, \lambda 2]}\left\{D_{\min -\exp [\lambda 1, \lambda 2]} / I_{(d \geq D \min -\exp [\lambda 1, \lambda 2])} \leq 1\right\} .
$$

Cette $\mathrm{D}_{\text {min-exp }}$ a été calculée pour 1 et 5 projecteurs de même type et de même puissance.

\section{Résultats}

\subsection{Répartition spectrale du rayonnement émis par les projecteurs}

La figure $3 a$ représente les répartitions spectrales relatives du rayonnement émis par les projecteurs équipés de lampes TH et HMI. Cette figure montre que, par rapport à la lampe $\mathrm{TH}$, la lampe $\mathrm{HMI}$ produit un rayonnement :

- ultraviolet et visible plus important ;

- infrarouge beaucoup plus faible. 


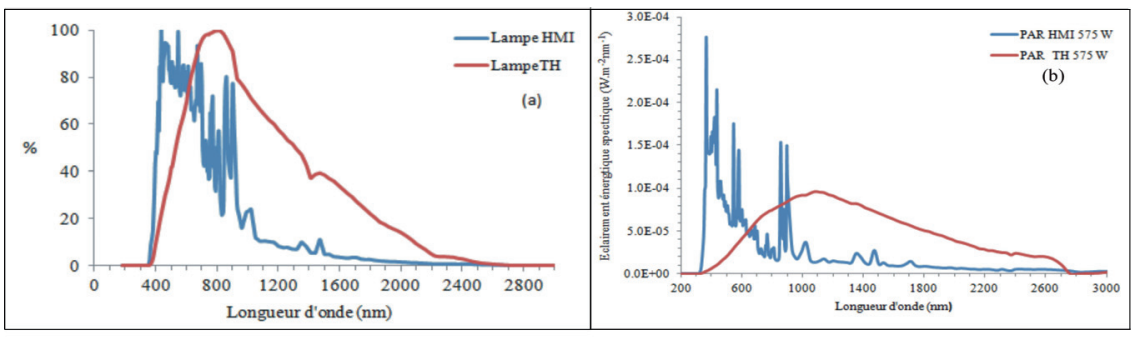

Figure 3 - Répartitions spectrales d'un projecteur PAR équipé de lampes TH et HMI de 575 W mesurées à $1,3 \mathrm{~m}$.

Spectral distribution of a PAR spotlight of $575 \mathrm{~W}$ with TH and HMI lamps measured at $1.3 \mathrm{~m}$.

À titre d'exemple, les spectres d'émission du rayonnement émis par un projecteur PAR équipé de lampes TH et HMI de $575 \mathrm{~W}$ sont représentés à la figure $3 \mathrm{~b}$. De cette figure, il ressort que le rapport du rayonnement émis par la lampe HMI à celui émis par la lampe TH est égal à 32 dans l'ultraviolet, à 2 dans le domaine de la lumière bleue et $1 / 3$ dans l'infrarouge.

Ces résultats permettent donc de soupçonner des risques prépondérants potentiels, dans les domaines ultraviolet et de la lumière bleue, pour les lampes HMI, et infrarouge pour les lampes TH.

\subsection{Répartition des projecteurs dans les différents groupes de risque définis par la norme EN 62417}

Dans un premier temps, afin de mettre en évidence le domaine spectral à l'origine du risque prépondérant, chaque projecteur a été classé dans un $\mathrm{GR}(\mathrm{x})$ pour chacun des domaines spectraux spécifiés comme suit :

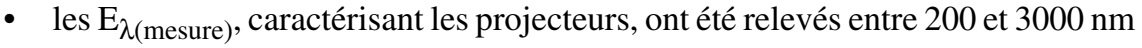
comme décrit au paragraphe 2.3.1;

- les $\mathrm{L}_{\mathrm{v}}$, de chaque projecteur et correspondant aux différents $\gamma_{\text {eff }}$ définis pour les différents GR(x) ( $c f$. Tab. V), ont été mesurés selon la méthode donnée au paragraphe 2.3.3;

- à partir de ces $\mathrm{E}_{\lambda \text { (mesure) }}$ et $\mathrm{L}_{\mathrm{v}}$ et pour un domaine spectral donné :

- les grandeurs énergétiques $\mathrm{E}_{\mathrm{S}}, \mathrm{E}_{\mathrm{UVA}}, \mathrm{L}_{\mathrm{B}}, \mathrm{L}_{\mathrm{R}}$ ou $\mathrm{E}_{\mathrm{IR}}$, selon le domaine spectral, définies dans le tableau III, ont été déterminées conformément aux méthodes décrites aux paragraphes 2.3.1 et 2.3.2;

- chaque grandeur a ensuite été comparée à la LE correspondante fixée pour les différents $\mathrm{GR}(\mathrm{x})(c f$. Tab. IV) ; 


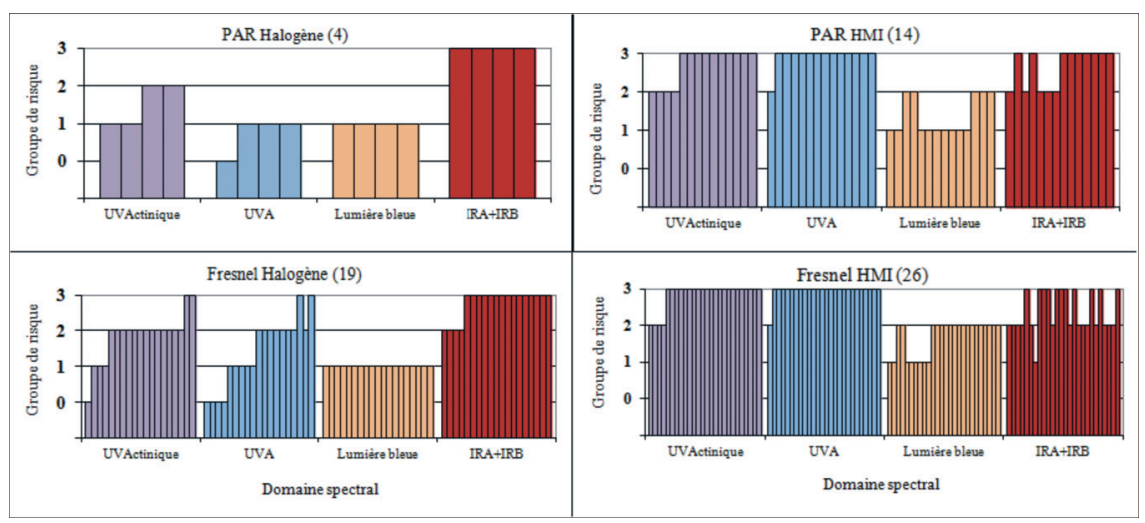

Figure 4 - Répartition des 63 projecteurs étudiés dans les $G R(x)$ correspondant aux différents domaines spectraux.

Distribution of the 63 spotlights studied in the GR(x) corresponding to the different spectral domains.

- si la grandeur énergétique précitée est inférieure à la LE du GR(x) considéré, alors ce GR(x) est affecté au projecteur ; sinon cette même grandeur énergétique est comparée à la LE du GR $(x+1)$ immédiatement supérieur et ainsi de suite.

Dans un second temps, un GR(x) a été affecté à chaque projecteur. Il s'agit du $\mathrm{GR}(\mathrm{x})$ d'ordre le plus élevé, sélectionné parmi ceux calculés précédemment pour chaque projecteur et pour les 5 domaines spectraux pris en compte.

La figure 4 représente la répartition des 63 projecteurs étudiés selon les GR(x) correspondant aux différents domaines spectraux pris en compte par la norme EN 62471. Toutefois, les $\mathrm{GR}(\mathrm{x})$ relatifs au domaine visible $+\mathrm{IR}_{\mathrm{A}}$, responsables de lésions thermiques rétiniennes, ne sont pas représentés sur ce graphique car ils sont toujours inférieurs aux $\mathrm{GR}(\mathrm{x})$ correspondant au domaine de la lumière bleue ; lesquels de ce fait ne peuvent pas être pris en considération pour déterminer les risques prépondérants.

De la figure 4 il ressort que :

- seul le type de lampes (TH ou HMI) a une influence sur les GR(x) des projecteurs ;

- dans le cas des projecteurs équipés d'une lampe TH :

- 19, sur les 23 testés, appartiennent au GR(3) dans le domaine $I_{A}+I_{B}$ $(780-3000 \mathrm{~nm})$; le risque prépondérant est donc celui dû au domaine $\mathrm{IR}_{\mathrm{A}}+$ $\mathrm{IR}_{\mathrm{B}}$;

- dans le domaine de la lumière bleue (300-700 nm), tous les projecteurs sont de GR(1) ; 
- en ce qui concerne les projecteurs munis d'une lampe HMI :

- 31, sur les 40 mesurés, appartiennent au GR(3) dans le domaine UV $\mathrm{Uctinique}_{\text {. }}$ $(200-400 \mathrm{~nm})$;

- 38 appartiennent au GR(3) dans le domaine $\mathrm{UV}_{\mathrm{A}}(315-400 \mathrm{~nm})$;

- 20 sont classés dans le GR(3) dans le domaine $\mathrm{IR}_{\mathrm{A}}+\mathrm{IR}_{\mathrm{B}}$;

- 22 sont de GR(2) dans le domaine de la lumière bleue (300-700 nm);

- les domaines spectraux $\mathrm{UV}_{\text {Actinique }}$ et $\mathrm{UV}_{\mathrm{A}}$ sont donc à l'origine des risques prépondérants.

En résumé, les domaines prépondérants du spectre sont donc les domaines $\mathrm{IR}_{\mathrm{A}}+$ $\mathrm{IR}_{\mathrm{B}}$, pour les lampes $\mathrm{TH}$, et $\mathrm{UV}_{\text {Actinique }}$ et $\mathrm{UV}_{\mathrm{A}}$, dans le cas des lampes HMI.

Au total 57 projecteurs sont de GR(3) et 6 de GR(2). Si on admet que tous les appareils à lampes de GR(2) et GR(3) sont dangereux pour les personnes qui y sont exposées, alors ces résultats montrent que tous les projecteurs testés présentent des risques certains pour les salariés du spectacle.

\subsection{Distances minimales d'exposition admissible $\left(D_{\text {min-exp }}\right)$ des projecteurs}

Les $\mathrm{D}_{\min -\exp }$ des différents projecteurs ont été déterminées, pour un projecteur seul et un groupe de 5 projecteurs de même type et de même puissance et pour $\mathrm{t}_{\max }=8 \mathrm{~h}$, selon la méthode décrite au paragraphe 2.4.

Une analyse de la variance à plusieurs facteurs a montré que seules les lampes ont un effet statistiquement significatif sur les $\mathrm{D}_{\text {min-exp }}:\left(\mathrm{F}_{262,47}=12,79 ; \mathrm{p}<0,01\right.$ pour un projecteur et $\mathrm{F}_{1978,32}=9,26 ; \mathrm{p}<0,01$ dans le cas d'un groupe de 5 projecteurs).

Les résultats des $\mathrm{D}_{\text {min-exp }}$ des lampes $\mathrm{TH}$ et HMI ainsi calculées sont présentés, en fonction de leur puissance, à la figure 5 .

Cette figure montre que :

- dans le cas des lampes TH de puissances comprises entre $300 \mathrm{~W}$ et $10 \mathrm{~kW}$ :

- pour un projecteur seul, les $\mathrm{D}_{\text {min-exp }}$ correspondantes varient de $0,80 \mathrm{~m}$ à $18,50 \mathrm{~m}$ et les domaines spectraux prépondérants qui les déterminent sont $\mathrm{I}^{\prime} I R_{\mathrm{A}}+\mathrm{IR}_{\mathrm{B}}$ et l'UV $\mathrm{A}$;

- pour un groupe de 5 projecteurs de même type et de même puissance, les $\mathrm{D}_{\text {min-exp }}$ correspondantes varient de $1,60 \mathrm{~m}$ à $41,70 \mathrm{~m}$ et les domaines spectraux prépondérants sont l'IR $\mathrm{A}_{\mathrm{A}}+\mathrm{IR}_{\mathrm{B}}$ et $\mathrm{l}^{\prime} \mathrm{UV} \mathrm{V}_{\mathrm{A}}$;

- il n'existe aucune relation directe entre $\mathrm{D}_{\text {min-exp }}$ et la puissance. En effet, à titre d'exemple les $\mathrm{D}_{\text {min-exp }}$ de projecteurs de puissances $2 \mathrm{~kW}$ et $10 \mathrm{~kW}$ sont respectivement égales à $18,40 \mathrm{~m}$ et $18,20 \mathrm{~m}$; 


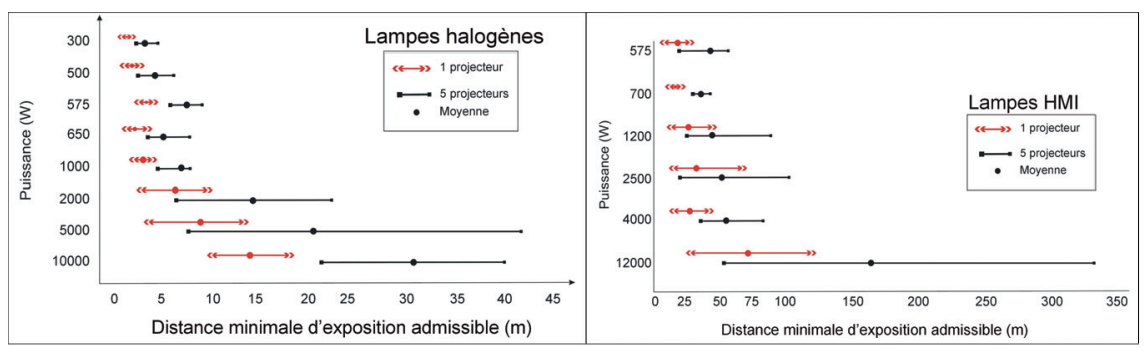

Figure 5 - $D_{\text {min-exp }}$ correspondant à un projecteur seul et un groupe de 5 projecteurs de même type et de même puissance pour un $t_{\text {max }}$ de $8 \mathrm{~h}$.

$D_{\text {min-exp }}$ corresponding to a single spotlight and a group of 5 spotlights of the same type and the same power for a $t_{\text {max }}$ of $8 \mathrm{~h}$.

- dans le cas des lampes HMI de puissances comprises entre $575 \mathrm{~W}$ et $12 \mathrm{~kW}$ :

○ pour un projecteur seul, les $\mathrm{D}_{\text {min-exp }}$ correspondantes varient de 2,30 $\mathrm{m}$ à $100 \mathrm{~m}$ et le domaine spectral prépondérant est l'UV $\mathrm{U}_{\mathrm{A}}$;

- pour un groupe de 5 projecteurs de même type et de même puissance, les $\mathrm{D}_{\text {min-exp }}$ correspondantes varient de $5,30 \mathrm{~m}$ à $320 \mathrm{~m}$ et les domaines spectraux prépondérants sont $\mathrm{l}^{\prime} \mathrm{IR}_{\mathrm{A}}+\mathrm{IR}_{\mathrm{B}}$ et $\mathrm{l}^{\prime} \mathrm{UV} \mathrm{A}$;

- comme précédemment, il n'existe aucune relation directe entre $D_{\min -\exp }$ et la puissance. À titre d'exemple, les $\mathrm{D}_{\text {min-exp }}$ de projecteurs de puissance $575 \mathrm{~W}$ et $2 \mathrm{~kW}$ sont respectivement égales à $8,30 \mathrm{~m}$ et $8,50 \mathrm{~m}$;

- les lampes HMI présentent des risques plus importants que les lampes TH, car quelle que soit la puissance, leurs $\mathrm{D}_{\text {min-exp }}$ respectives sont toujours supérieures à celles des lampes TH.

\section{Discussion}

\subsection{Classification des projecteurs dans les différents $G R(x)$ définis par la norme EN 62471}

Les 63 projecteurs étudiés ont été répartis dans les 4 GR(x), définis par la norme EN 62471, selon la méthode décrite au paragraphe 2.2, comme indiqué dans le tableau VI.

Ce tableau montre que tous les projecteurs étudiés, appartiennent aux GR(2) et GR(3) ; ce qui signifie qu'ils présentent des risques majeurs dans les domaines spectraux :

- $\quad \mathrm{IR}_{\mathrm{A}}+\mathrm{IR}_{\mathrm{B}}(780-3000 \mathrm{~nm})$ dans le cas des lampes TH ; ce domaine spectral est responsable d'affections à effets retardés tels que les opacifications lenticulaires (cataractes) (ICNIRP, 1997); 
TABLEAU VI

Répartition des projecteurs dans les différents GR(x).

Distribution of spotlights in the different GR(x).

\begin{tabular}{cccc}
\hline & Type & \multicolumn{2}{c}{ Nombre de projecteurs } \\
\hline \multirow{2}{*}{ Projecteur } & Lampe & GR(2) & GR(3) \\
FRESNEL & TH & 4 & 15 \\
\cline { 2 - 4 } & HMI & 1 & 25 \\
PAR & TH & - & 4 \\
\cline { 2 - 4 } & HMI & 1 & 13 \\
TOTAL & 63 & 6 & 57 \\
\hline
\end{tabular}

- $\mathrm{UV}_{\text {Actinique }}(200-400 \mathrm{~nm})$ et $\mathrm{UV}_{\mathrm{A}}(315-400 \mathrm{~nm})$ pour les lampes HMI ; lesquels sont susceptibles d'induire des affections (ICNIRP, 2004 ; Césarini, 2007):

- oculaires aigües telles que des inflammations de la cornée (photokératite) et de la conjonctive (photoconjonctivite), affections qui s'associent en pathologie professionnelle sous le vocable de « coup d'arc du soudeur », ou cutanées aigües, qui vont du simple érythème jusqu'à des réponses lésionnelles plus importantes, œdèmes, phlyctènes ;

- oculaires retardées telles que cataractes et cutanées retardées, vieillissement, élastose, carcinomes et mélanomes.

Les résultats présentés ci-dessus montrent la nécessité de protéger les salariés des arts et du spectacle en réduisant les risques à des niveaux admissibles.

L'utilisation de lampe HMI double enveloppe (traitée anti-UV) ou de filtres « anti-UV » placés devant la surface d'émission des projecteurs peut permettre de réduire d'un facteur 100 le rayonnement $U_{C}+U_{B}(200-315 \mathrm{~nm})$ et d'un facteur 4 le rayonnement $\mathrm{UV}_{\mathrm{A}}$ produits par la lampe. De plus, le maquillage, les crèmes « antivieillissement » ou l'utilisation de crèmes solaires dites « waterproof » sont susceptibles de protéger efficacement la peau des artistes durant le spectacle (O'Hagan et al., 2011). En revanche, les risques oculaires inhérents à l' $\mathrm{IR}_{\mathrm{A}}+\mathrm{IR}_{\mathrm{B}}$ ne peuvent être réduits dans de grandes proportions que par l'utilisation de filtres dopés à l'aide d'oxydes métalliques et placés à l'avant du projecteur. Cependant, ces filtres, bien qu'efficaces, ont l'inconvénient d'absorber une grande partie du rayonnement visible et de diffuser une lumière verdâtre, donc de changer la température de couleur des lampes ; ce qui contrarie les règles d'esthétique appliquées par les éclairagistes.

Utilisé dans le cadre de l'éclairage scénique, le schéma de classification, proposé par la norme EN 62471 permet, lors de l'élaboration d'un «plan lumière », de sélectionner les projecteurs susceptibles de présenter des risques 
mineurs appartenant aux GR(0) et GR(1). Or, l'éclairage d'une scène utilise généralement entre 50 et 300 appareils (voire plus) selon le type de spectacle; ce grand nombre de projecteurs, même de $\mathrm{GR}(0)$, peut induire des risques non négligeables.

Cette classification n'est donc pas suffisante pour évaluer les risques dus à un «plan lumière». L'utilisation d'outil de simulation tel que «CatRayon ${ }^{\circledR}$ », s'avère un complément indispensable.

\subsection{Distances minimales d'exposition admissibles ( $\left.D_{\text {min-exp }}\right)$ des projecteurs}

Les $\mathrm{D}_{\text {min-exp }}$, déterminées pour chaque projecteur varient selon leur puissance et quel que soit le type de lampe, de $0,80 \mathrm{~m}$ à $100 \mathrm{~m}$. Comme, selon le type de spectacle, des groupes de 50 à 300 projecteurs sont susceptibles d'être mis en œuvre, alors, dans ces conditions, les $\mathrm{D}_{\min -\exp }$ qui leur correspondent sont respectivement comprises entre 5,60 et $710 \mathrm{~m}$ et 14,00 et 1730,00 m environ. Ces distances sont incompatibles avec les dimensions des scènes de spectacle, qui à notre connaissance, peuvent atteindre $30 \mathrm{~m} \times 25 \mathrm{~m}$. Il en est de même pour $\mathrm{t}_{\max }$. En effet, si les $\mathrm{D}_{\text {min-exp }}$, calculées pour 1 projecteur sont appliquées à des ensembles de 50 et 300 projecteurs de même type, les $t_{\max }$ deviennent alors respectivement égales à 9 min $30 \mathrm{~s}$ et $1 \mathrm{~min} 30 \mathrm{~s}$ par journée de travail. Ces durées maximales d'exposition journalière ne sont pas réalistes au regard des durées des spectacles habituellement rencontrées, à savoir de $1 \mathrm{~h} 30$ à $2 \mathrm{~h} 30$.

La distance d'exposition et la durée d'exposition journalière aux projecteurs scéniques ne constituent donc pas des paramètres pertinents de réduction des risques. Les projecteurs scéniques traditionnels à lampe posent donc un réel problème pour la santé des salariés des arts du spectacle.

Néanmoins, de tels projecteurs dont la lampe (TH ou HMI) a été remplacée par un pavé de LEDs, ont été mis sur le marché récemment. Il s'agit de projecteurs dotés de toutes les caractéristiques optiques et fonctionnelles des projecteurs à lampe. Ces projecteurs présentent l'avantage de n'émettre aucun rayonnement ultraviolet et infrarouge. Par conséquent, ils ne présentent, à l'évidence, aucun risque dans ces domaines spectraux et seul le risque de lésions photochimiques rétiniennes, lié à la lumière bleue, persiste (Césarini, 2009). À titre d'exemple, un projecteur à LEDs, de puissances 75 W est de GR(1), selon la norme EN 62471. Ces projecteurs pourraient se présenter comme une alternative aux projecteurs scéniques traditionnels à lampe. Toutefois, il conviendra de réaliser une étude afin de vérifier si leur niveau de risque dans le domaine de la lumière bleue est acceptable ou non. 


\section{Conclusion}

Cette étude a mis en évidence que les projecteurs scéniques sont susceptibles de poser des problèmes pour la santé des salariés des arts du spectacle. En effet, les 63 projecteurs étudiés présentent des risques certains ; ils sont, pour 57 d'entre eux, classés GR(3) et pour les 6 autres GR(2), selon la norme EN 62471. Toutefois cette norme ne concerne qu'un seul projecteur ; elle ne permet donc que de sélectionner les appareils d'éclairage sans risque ou à risque faible, respectivement de $\mathrm{GR}(0)$ ou GR(1), pour élaborer un «plan lumière ». Or, ce dernier peut être constitué de plusieurs centaines de projecteurs de GR(0) ; lesquels peuvent induire des risques non négligeables. Le schéma de classification proposé par ladite norme n'est donc pas suffisant pour évaluer les risques associés à un «plan lumière » composé de projecteurs traditionnels à lampe ; l'utilisation d'outil de simulation s'avère un complément indispensable.

De plus, la distance d'exposition et la durée d'exposition journalière aux projecteurs scéniques ne constituent pas des paramètres pertinents pour réduire les risques à des valeurs admissibles.

Toutefois, des projecteurs récemment commercialisés, dont la lampe a été remplacée par un pavé de LEDs pourraient constituer une alternative aux projecteurs scéniques traditionnels à lampe. Il conviendra, toutefois, de vérifier si une exposition chronique à la lumière bleue qu'ils émettent n'est pas nocive pour la rétine.

Remerciements. Les auteurs remercient les sociétés ADB Lighting Technologies, Magnum et Robert Juliat, France Télévision, l'Opéra National de Paris, l'Association des Producteurs de Cinéma et le Comité Central d'Hygiène et de Sécurité de la production Cinématographique d'avoir mis grâcieusement à leur disposition les projecteurs nécessaires à cette étude.

\section{RÉFÉRENCES}

Barlier-Salsi A., Salsi S. (1996) Eclairement énergétique produit par une source de rayonnement optique plane de forme complexe dans une direction quelconque - Calcul du facteur de configuration par la méthode des éléments finis, Les notes scientifiques et techniques de l'INRS, $\mathrm{N}^{\circ} 140,62$ pages.

Barlier-Salsi A., Salsi S. (2007a) CatRayon3 : Un logiciel pour évaluer l'exposition au rayonnement optique dans les locaux de travail et déterminer les moyens de protection, Les notes scientifiques et techniques de l'INRS, $\mathrm{N}^{\circ} 265,57$ pages.

Barlier-Salsi A., Salsi S. (2007b) Evaluation de l'exposition aux rayonnements optiques dans les locaux de travail et détermination des moyens de protection avec CatRayon 3, Hygiène et Sécurité du Travail, ND 2270, 15 pages. 
CEI/TR 62471-2 (2009) Photobiological safety of lamps and lamp systems - Part 2: Guidance on manufacturing requirements relating to non-laser optical radiation safety, 45 pages.

Césarini J.P. (2007) Rayonnement ultraviolet et santé, Radioprotection 42 (3), 379-392.

Césarini J.P. (2009) Risques oculaires du rayonnement bleu, Radioprotection 44 (4), 463-478.

Hietanen M.T.K., Hoikkala M. (1990) Ultraviolet Radiation and Blue Light from Photofloods in Television Studios and Theaters, Health Phys. 59 (2), 193-198.

ICNIRP (1997) Guidelines on limits of exposure to broad-band incoherent optical radiation (0.38 to $3 \mu \mathrm{m})$, Health Phys. 73 (3), 539-554.

ICNIRP (2004) Guidelines on limits of exposure to ultraviolet radiation of wavelenghts $180 \mathrm{~nm}$ to $400 \mathrm{~nm}$ (incoherent optical radiation), Health Phys. 87 (2), 171-186.

INRS (2012) CatRayon 4, logiciel d'évaluation de l'exposition aux rayonnements optiques dans les locaux de travail (Téléchargement, Downloading), http://www.inrs.fr/accueil/produits/ mediatheque/doc/outils.html?refINRS=outil03.

Norme EN 62471 (2008, September) Sécurité photobiologique des lampes et appareils utilisant des lampes. CENELEC, Bruxelles, 46 pages.

O’Hagan J.B., Khazova M., Jones C.W. (2011) Ultra-violet emission from HMI daylight luminaires. Lighting Res. Technol. 43, 249-257.

Parlement européen (2006) Directive 2006/25/CE du parlement européen et du conseil, du 5 avril 2006, relative aux prescriptions minimales de sécurité et de santé relatives à l'exposition des travailleurs aux risques dus aux agents physiques (rayonnements optiques artificiels), Journal Officiel, 38-59.

Schulmeister K. (2001) Concepts in dosimetry related to laser safety and optical radiation hazard evaluation. Dans SPIE proceeding: Laser and Noncoherent Light Ocular Effects: Epidemiology Prevention and Treatment 4246, 105-116.

Schulmeister K., Stuck B., Lund D.J., Sliney D. (2011) Review of thresholds and recommandations for revised exposure limits for laser and optical radiation for thermally induced retinal injury, Health Phys. 100, 210-220.

\section{ANNEXE \\ Symboles, termes et unités}

$\mathrm{TH}$ :

Lampe halogène.

HMI :

Lampe aux halogénures métalliques.

IRC :

Indice de rendu des couleurs (sans dimension).

$\lambda$ :

Longueur d'onde (nm).

$\mathrm{E}_{\lambda}$ :

Eclairement énergétique spectrique $\left(\mathrm{W} \cdot \mathrm{m}^{-2} \cdot \mathrm{nm}^{-1}\right)$.

$\mathrm{L}_{\lambda}$ :

Luminance énergétique spectrique (W. $\left.\mathrm{m}^{-2} \cdot \mathrm{nm}^{-1} \cdot \mathrm{sr}^{-1}\right)$.

$\operatorname{Le}_{[\lambda 1, \lambda 2]}:$

Luminance énergétique correspondant au domaine spectral de longueurs d'onde comprises ente $\lambda 1$ et $\lambda 2$ $\left(\mathrm{W} \cdot \mathrm{m}^{-2} \cdot \mathrm{nm}^{-1}\right)$.

$\Delta \lambda: \quad$ Largeur de bande (nm). 
$\mathrm{S}(\lambda):$

$\mathrm{B}(\lambda)$ :

$R(\lambda):$

LE :

$\mathrm{LE}_{[\lambda 1, \lambda 2]}:$

$\mathrm{E}_{\mathrm{S}}:$

$\mathrm{E}_{\mathrm{UVA}}$ :

$\mathrm{L}_{\mathrm{B}}$ :

$\mathrm{L}_{\mathrm{R}}$ :

$\mathrm{E}_{\mathrm{IR}}$ :

$\gamma_{\text {eff }}$ :

$\mathrm{L}_{\mathrm{v}}$ :

$\mathrm{E}_{\mathrm{v}}$ :

$t_{\max }:$
Pondération spectrale qui tient compte du rapport entre la longueur d'onde et les effets du rayonnement ultraviolet sur les yeux et la peau (sans dimension).

Pondération spectrale qui tient compte du rapport entre la longueur d'onde et la lésion photochimique de l'œil provoquée par la lumière bleue (sans dimension).

Pondération spectrale qui tient compte du rapport entre la longueur d'onde et la lésion de l'œil par effet thermique provoquée par des rayonnements visibles et IRA (sans dimension).

Limites d'exposition spécifiées par la norme EN 62471 dans les différents domaines spectraux (W. $\left.\mathrm{m}^{-2} \mathrm{ou} \mathrm{W} \cdot \mathrm{m}^{-2} \cdot \mathrm{sr}^{-1}\right)$.

Limites d'exposition spécifiées par la norme EN 62471 dans le domaine spectral de longueurs d'onde comprises entre $\lambda 1$ et $\lambda 2\left(\mathrm{~W} . \mathrm{m}^{-2}\right.$ ou W.m $\left.{ }^{-2} \cdot \mathrm{sr}^{-1}\right)$.

Eclairement énergétique efficace $\left(\mathrm{UV}_{\text {Actinique }}\right)$, calculé sur le domaine spectral $180-400 \mathrm{~nm}$ et pondéré en fonction de la longueur d'onde $\operatorname{par} S(\lambda)\left(\mathrm{W} \cdot \mathrm{m}^{-2}\right)$.

Eclairement énergétique total $\left(\mathrm{UV}_{\mathrm{A}}\right)$, calculé sur le domaine spectral 315-400 nm (W.m $\left.{ }^{-2}\right)$.

Luminance énergétique efficace (lumière bleue), calculée sur le domaine spectral 300-700 nm et pondérée en fonction de la longueur d'onde par $\mathrm{B}(\lambda)\left(\mathrm{W} \cdot \mathrm{m}^{-2} \cdot \mathrm{sr}^{-1}\right)$.

Luminance énergétique efficace (lésion par effet thermique), calculée sur le domaine spectral 380$1400 \mathrm{~nm}$ ou 780-1400 $\mathrm{nm}$ et pondérée en fonction de la longueur d'onde par $\mathrm{R}(\lambda)\left(\mathrm{W} \cdot \mathrm{m}^{-2} \cdot \mathrm{sr}^{-1}\right)$.

Eclairement énergétique total (lésion par effet thermique), calculé sur le domaine spectral 780-3000 nm $\left(\mathrm{W} . \mathrm{m}^{-2}\right)$.

Champ de vision effectif selon la valeur des $t_{\max }$ spécifiées pour les différents groupes de risques ( $\mathrm{Rad})$.

Luminance lumineuse mesurée dans le champ de vision effectif $\gamma_{\text {eff }}\left(\mathrm{Cd} \cdot \mathrm{m}^{-2}\right)$.

Eclairement lumineux produit par les projecteurs à $0,20 \mathrm{~m}$, il se détermine à partir de $\mathrm{E}_{\lambda}$ et $\mathrm{V}(\lambda)$ (lux).

Durée maximale d'exposition journalière admissible spécifiée pour les différents groupes de risques (S). 
$\alpha_{\text {projecteur }}:$

$\mathrm{R}$ :

$\tau_{\text {oculaire }}:$

$\mathrm{D}_{\text {max-exp }}$ :

$\mathrm{D}_{\max -\exp [\lambda 1, \lambda 2]}$ :

$\mathrm{F}_{\text {config }}$ :

$\mathrm{F}_{\text {config }(0,20 \mathrm{~m})}$ :

$\mathrm{F}_{\text {config(mesure) }}$ :

$\mathrm{F}_{\text {config }(\operatorname{Dmin}-\exp [\lambda 1, \lambda 2])}$

$\mathrm{GR}(\mathrm{x})$ :

$[\lambda 1, \lambda 2]:$

$\mathrm{I}_{(0,20 \mathrm{~m}[\lambda 1, \lambda 2])}:$

$\mathrm{I}_{(\mathrm{Dmin}-\exp [\lambda 1, \lambda 2])}:$
Dimension angulaire propre du projecteur vue de l'observateur (Rad).

Rayon de la surface d'émission des projecteurs (m).

Facteur de transmission des milieux oculaires (sans dimension).

Distance minimale d'exposition admissible (m).

Distance minimale d'exposition admissible correspondant au domaine spectral $[\lambda 1, \lambda 2](\mathrm{m})$.

Le facteur de configuration du faisceau de rayonnement reliant un élément de surface de la source (ds) et un élément de surface du récepteur (ds') distant de $\mathrm{D}$ a pour expression :

$$
d^{2} G=\frac{d s \cdot d s^{\prime} \cdot \cos \theta_{s} \cdot \cos \theta_{s^{\prime}}}{D^{2}} \text { et } G=\int_{s} d^{2} G
$$

Facteur de configuration d'un projecteur correspondant à une distance de 0,20 m (sr).

Facteur de configuration d'un projecteur correspondant aux conditions de mesures (sr).

: Facteur de configuration d'un projecteur correspondant à la distance de $\mathrm{D}_{\text {min-exp }[\lambda 1, \lambda 2]}$ (sr).

Groupe de risque (0, 1, 2, ou 3) selon la norme EN 62471 (sans dimension).

Domaine spectral de longueurs d'onde comprises entre $\lambda 1$ et $\lambda 2(\mathrm{~nm})$.

Indice de risque relatif au domaine de longueurs d'onde comprises entre $\lambda 1$ et $\lambda 2$ calculé pour une distance d'exposition de $0,20 \mathrm{~m}$ (sans dimension).

Indice de risque déterminé pour la distance minimale d'exposition admissible du domaine spectral de longueurs d'onde comprises entre $\lambda 1$ et $\lambda 2$ (sans dimension). 